\title{
Hybrid supercapacitor-battery materials for fast electrochemical charge storage
}

SUBJECT AREAS:

NANOCOMPOSITES

BATTERIES

KINETICS AND DYNAMICS

POLYMERS

Received

15 January 2014

Accepted

20 February 2014

Published

7 March 2014

Correspondence and requests for materials should be addressed to

S.M. (sorin.melinte@ uclouvain.be); P.M.A. (ajayan@rice.edu) or J.-F.G. (jean-francois. gohy@uclouvain.be)

\author{
A. Vlad', N. Singh'2, J. Rolland ${ }^{3}$, S. Melinte', P. M. Ajayan ${ }^{2}$ \& J.-F. Gohy ${ }^{3}$
}

'Institute of Information and Communication Technologies, Electronics and Applied Mathematics, Electrical Engineering, Université catholique de Louvain, Louvain la Neuve, B-1348 Belgium, ${ }^{2}$ Department of Mechanical Engineering and Materials Science, Rice University, Houston, Texas 77005, United States, ${ }^{3}$ Institute of Condensed Matter and Nanosciences, Bio-and Soft Matter, Université catholique de Louvain, Louvain la Neuve, B-1348 Belgium.

High energy and high power electrochemical energy storage devices rely on different fundamental working principles - bulk vs. surface ion diffusion and electron conduction. Meeting both characteristics within a single or a pair of materials has been under intense investigations yet, severely hindered by intrinsic materials limitations. Here, we provide a solution to this issue and present an approach to design high energy and high power battery electrodes by hybridizing a nitroxide-polymer redox supercapacitor (PTMA) with a Li-ion battery material $\left(\mathrm{LiFePO}_{4}\right)$. The PTMA constituent dominates the hybrid battery charge process and postpones the $\mathrm{LiFePO}_{4}$ voltage rise by virtue of its ultra-fast electrochemical response and higher working potential. We detail on a unique sequential charging mechanism in the hybrid electrode: PTMA undergoes oxidation to form high-potential redox species, which subsequently relax and charge the $\mathrm{LiFePO}_{4}$ by an internal charge transfer process. A rate capability equivalent to full battery recharge in less than 5 minutes is demonstrated. As a result of hybrid's components synergy, enhanced power and energy density as well as superior cycling stability are obtained, otherwise difficult to achieve from separate constituents.

i-ion batteries (LIBs) with high specific energy, high power density, long cycle life, low cost and high margin of safety are critical for widespread adoption of electric vehicles $(\mathrm{EVs})^{1-8}$. A key bottleneck in achieving this goal is the limited fast charging ability of LIBs. Rapid charging causes accelerated degradation of the battery constituents as well as a potential fire hazard due to local over-potential build-up and increased heat generation $^{9-12}$. LIBs have the highest energy density but typically suffer from low power by virtue of reversible Coulombic reactions occurring at both electrodes, involving charge transfer and ion diffusion in bulk electrode materials ${ }^{13}$. Since both diffusion and charge transfer are slow processes, power delivery as well as the recharging time of the LIBs are kinetically limited. On the other extreme, electrochemical double-layer supercapacitors (EDLCs), which store energy through accumulation of ions on the electrode surface, have low energy storage capacity but very high power density ${ }^{14}$.

A special category of electrochemical capacitors is provided by redox capacitors (REC), which usually make use of electroactive polymers or transition metal oxides ${ }^{15-17}$. Here, charge is stored through surface or bulk (pseudocapacitive) redox reactions, similar to LIB materials, yet, with a very fast charge transfer response, similar to EDLCs. Although excellent capacity retention for extended cycling can be obtained even at high charge discharge rates ${ }^{14,17}$, specific capacity of RECs is typically lower than for LIBs. Moreover, especially in the case of electroactive polymer based supercapacitors, excess electrolyte has to be used to compensate the electrolyte depletion, diminishing the gravimetric capacity.

The most intuitive approach to combine high energy and high power density within a single device is to combine the different types of energy storage sources. So far, mainly hybridization between EDLCs and LIBs has been explored ${ }^{18}$. The electrochemical response of such hybrids has been found to be the sum of the responses of the separate components and is schematically depicted in Figure $1 a^{19-22}$. The contribution of components to the total stored charge is proportional to the amount of each component, whereas the electrode configuration and composition control the power and energy delivery performances ${ }^{18}$. The primary drawback of this approach is that power and energy performances are decoupled. At high current densities, the response is dominated by the EDLC component, considerably diminishing the energy density of the hybrid device. Utmost, the sloping potential profile of the EDLC component in the electrochemical response is detrimental for most of the applications where a constant power supply is required. To overcome these limitations, a hybrid electrode, which makes synergistic use of capacitor and battery components rather than a merely additive composite, is necessary. 


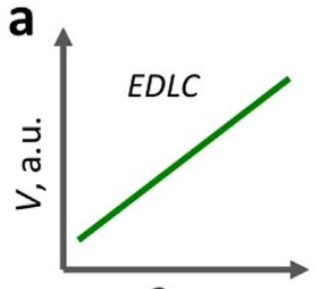

Q, a.u.

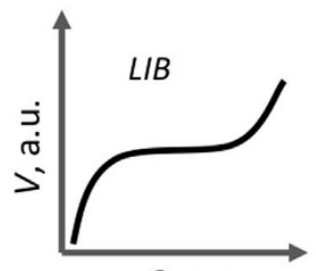

Q, a.u.
Traditional Hybridization

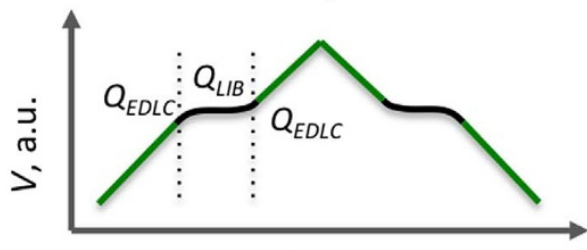

Q, a.u.

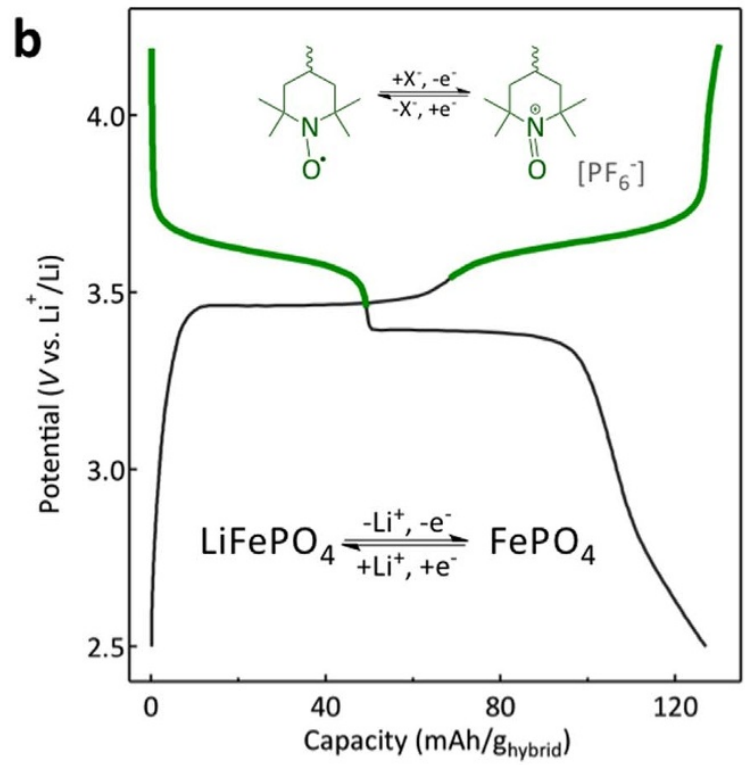

Figure 1 Hybridization principle and materials. (a), Voltage vs. capacity profiles for typical capacitor and battery materials as well as for traditional hybridization. The stored charge $(Q)$ is low given the low specific capacity of the capacitive component and the LIB plateau vanishes at high chargedischarge rates. (b), Voltage profile of the hybrid PTMA-LiFePO 4 electrode at a current density of $26 \mathrm{mAh} / \mathrm{g}_{\text {hybrid }}$. Both plateaus are discernible in charge and discharge and correspond to distinct redox processes of PTMA and $\mathrm{LiFePO}_{4}$. For constituents mass ratio of 1.6, the capacity contribution ratio of each component is $\sim 1$, corresponding to a specific capacity of $\sim 126 \mathrm{mAh} / \mathrm{g}_{\text {hybrid. }}$.

While attempts in this direction have been proposed, unfavorable choice of components or overlap of redox couples has prevented complete realization of a true hybrid electrode ${ }^{23,24}$. The redox mediators, also known as redox shuttle systems, can conceptually provide a valuable solution for the above-described limitation ${ }^{25,26}$. Usually, these systems are composed of small redox molecules, solubilized in the electrolyte or grafted on the surface of active material, capable of rapid charge transfer and have been primarily used for electrode overcharge protection ${ }^{27-30}$. However, the redox shuttle has a little contribution to the stored charge and there is little knowledge of its effect on the enhancement of the electrode rate performance.

Here, we show that enhanced battery-capacitor hybrids can be constructed by careful choice of the super-capacitor and battery components. To materialize this idea, we hybridized lithium iron phosphate $\left(\mathrm{LiFePO}_{4}\right)$ battery material with poly $(2,2,6,6$-tetramethyl-1-piperinidyloxy-4-yl methacrylate) (PTMA) redox capacitor. The hybrid electrode displays two distinct charge - discharge plateaus consistent with redox processes in $\mathrm{LiFePO}_{4}$ and PTMA constituents (Fig. 1b). We show that the excellent synergy between the PTMA and LiFePO4 gives best-of-both-worlds performance characteristics: high energy and power capacity, over-polarization protection, unique input power-leveling capability as well as fast and stable recharge for more than 1,500 cycles. The key design principle behind the hybrid battery concept is rapid electrochemical response combined with higher working potential of PTMA.

Hybrid design and electrochemical performance. PTMA is commonly used as the main component of the organic radical battery due to the radical nature of the charge storing nitroxide group ${ }^{31}$. Its theoretical specific capacity is $111 \mathrm{mAh} / \mathrm{g}$ with a flat-potential profile response at an average voltage of $3.6 \mathrm{~V} v s$. $\mathrm{Li}^{\prime} / \mathrm{Li}^{+}$(Fig. 1b). Electron transfer kinetics of the nitroxide radical has been measured as high as $10^{-1} \mathrm{~cm} / \mathrm{s}$, resulting in ultra-fast charge transfer within the radical polymer layer ${ }^{32,33}$. The PTMA used in this study displays long cycle lifetime, ultra-fast rate capability and low electrode polarization at elevated rates (supplementary information: materials and methods and Figs. S1-S6). In contrast, $\mathrm{LiFePO}_{4}$ is a crystalline inorganic material with limited bulk electrical conductivity and anisotropic
$\mathrm{Li}^{+}$diffusivity ${ }^{34-37}$. Its high theoretical specific capacity of 170 $\mathrm{mAh} / \mathrm{g}$, flat $\mathrm{Li}^{+}$de/intercalation plateau potential at $3.4 \mathrm{~V} v s$. Li/ $\mathrm{Li}^{+}$(Fig. 1b) and the abundance of the constituent materials has made $\mathrm{LiFePO}_{4}$ one of the most sought after battery material for future $\mathrm{EV} \mathrm{s}^{38,39}$. While high power $\mathrm{LiFePO}_{4}$ can be designed by properly tailoring the size and the crystallinity of the particles, adding surface carbon coating or excess conductive additives as well as by molecular doping ${ }^{40}$, intrinsically, PTMA still displays much higher power performances due to its ultrafast electron transfer kinetics and electrolyte swelled gel-like morphology allowing for high ionic diffusivity ${ }^{33,41-43}$. We expect that proportionally enhanced performances of the hybrid $\mathrm{LiFePO}_{4}$-PTMA configuration will be obtained by improving the power capabilities of separate or both components.

The cyclic voltammetry response of the hybrid electrode is presented in Figure 2a (supplementary information: Fig. S7 shows the full scan rate range). Two sets of distinct peaks (both, in reduction and oxidation) are clearly visible at low scan rates, consistent with the charge - discharge plateaus observed in the galvanostatic cycling (Fig. 1b). At higher scan rates, however, different behavior is observed for anodic and cathodic processes. Figure $2 \mathrm{~b}$ shows the cathodic peaks positions as a function of the scan rate for the hybrid electrode. While the separation between the anodic voltages increases (Fig. 2a, see also supplementary information: Fig. S8), the two cathodic peaks merge into a single peak, dominated by the PTMA cathodic process. An electrode based only on $\mathrm{LiFePO}_{4}$ will reach unsafe levels of voltage polarization even at moderate scan rates (dashed curve in Fig. 2b; see also supplementary information: Fig. S9 for single constituent electrode characterization). In contrast, the cathodic polarization of the hybrid electrode is significantly lower than that of a pure $\mathrm{LiFePO}_{4}$ electrode, especially after the $\mathrm{LiFePO}_{4}$ and PTMA cathodic processes merge around $0.8 \mathrm{mV} / \mathrm{s}$. It should be noted that even if the $\mathrm{LiFePO}_{4}$ cathodic peak cannot be perceived, $\mathrm{LiFePO}_{4}$ undergoes the oxidation process at high scan rates. This is fundamentally different from the single $\mathrm{LiFePO}_{4}$ constituent electrode configuration where the fast polarization increase will drive the electrode potential outside the safe voltage range preventing the redox reaction. This gives a significant advantage to the hybrid 


\section{a}

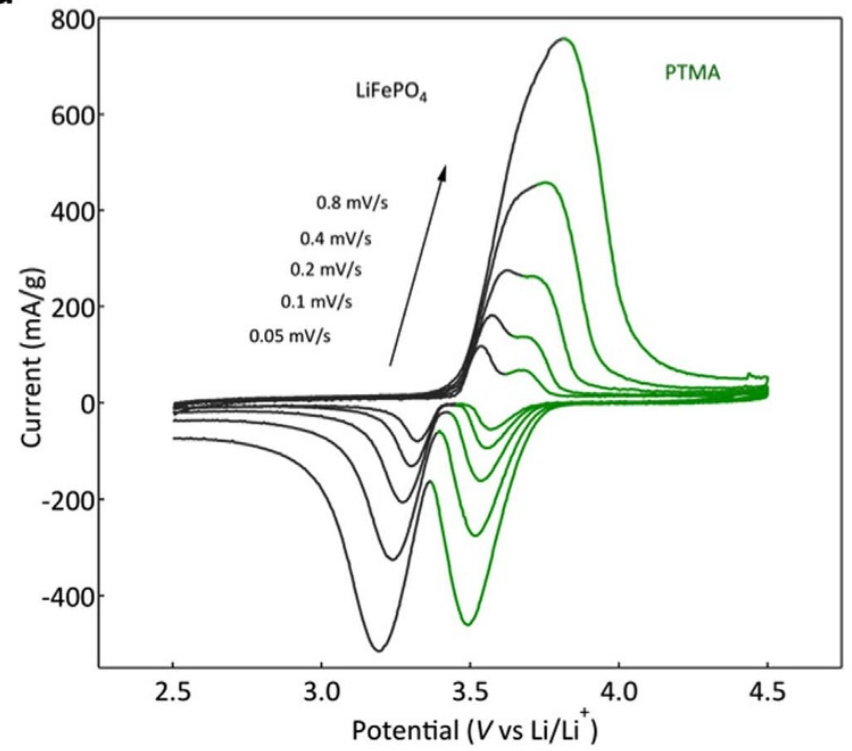

b
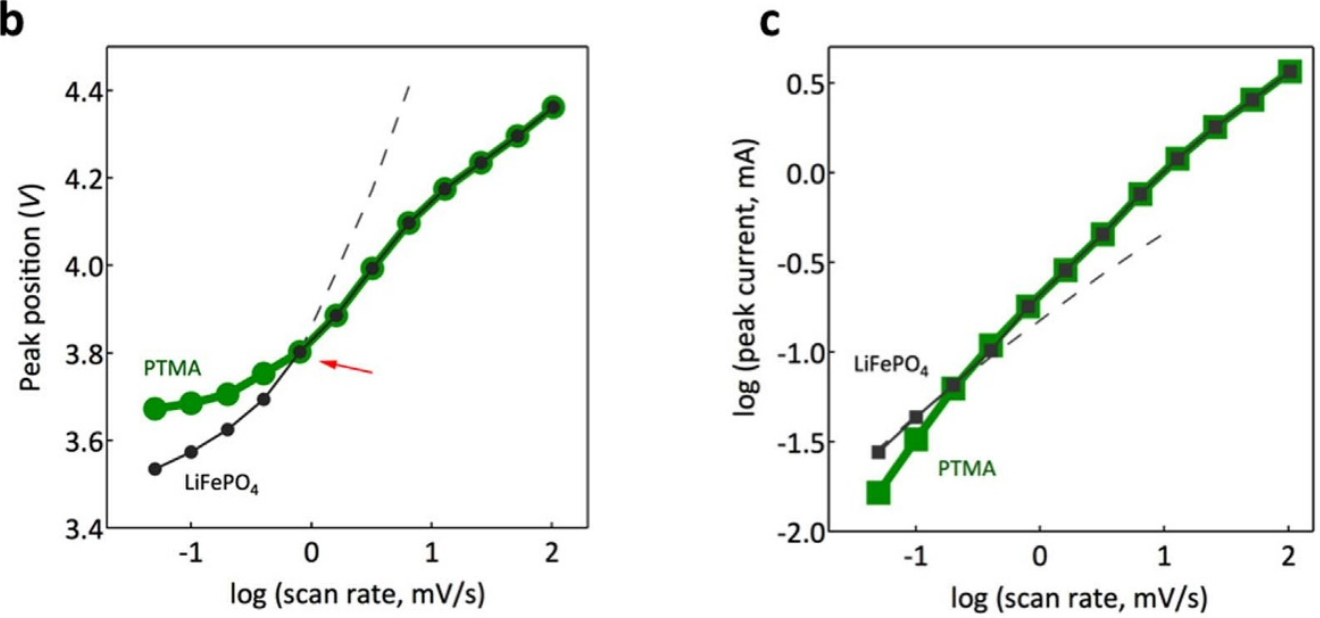

Figure $2 \mid$ Cyclic voltammetry of the hybrid electrode. (a), Voltammograms recorded between 2.5 and $4.5 \mathrm{~V}$ vs. $\mathrm{Li} / \mathrm{Li}^{+}$at different scan rates. The two cathodic peaks are separated at low scan rates and overlap at higher scan rates. (b), Cathodic peaks position function of the scan rate for the hybrid electrode. Red arrow marks the onset of the overlapping region. No distinct $\mathrm{LiFePO}_{4}$ redox peak is observed beyond. (c), Cathodic peak current function of the scan rate for the hybrid electrode. The slope of the log-log dependence is indicative of the charge transfer kinetics. The dashed curves in (b), and (c), are extrapolated from the voltammetry scan of the $\mathrm{LiFePO}_{4}$ electrode (see SI).

configuration: the overall electrode potential being limited by PTMA, avoids the voltage abuse on $\mathrm{LiFePO}_{4}$ particles accentuated at high rates, ultimately circumventing the degradation of the $\mathrm{LiFePO}_{4}{ }^{44}$.

The relationship between the peak current intensity and scan rates provides insight into the electrode redox kinetics. Figure $2 \mathrm{c}$ shows the $\log (i)$ versus $\log (v)$ plot for the hybrid electrode where $i$ is the peak current and $v$ is the scan rate. The current follows a power law dependence function of scan rate $i \sim v^{p}$. The power coefficient $p$ is indicative of the charge storage kinetics in the electrode ${ }^{45}$. The PTMA cathodic current shows a linear dependence $(p \sim 1)$ indicative of very fast, surface controlled kinetics corroborating the high counter-ion mobility as well as the ultra-fast electron transfer kinetics of the nitroxide radical ${ }^{4,32,33}$. At higher scan rates, a slow deviation is observed mainly attributed to the ohmic contributions (supplementary information: Fig. S8). In turn, $\mathrm{LiFePO}_{4}$ displays diffusion-controlled response $(p \sim 0.6)$, further limited at higher scan rates (supplementary information: Fig. S8). In the hybrid electrode, both constituents follow their intrinsic behavior prior the convergence point while after, the charge storage kinetics is as well dominated by the PTMA constituent. This highlights one of the fundamental characteristics of the hybrid electrode: at high scan rates or, in other words, under fast redox kinetics, the cathodic process (polarization and charge transfer rate) is exclusively dominated by the PTMA redox. It should be mentioned that the intrinsic properties of the used $\mathrm{LiFePO}_{4}$ are not being modified in the hybrid configuration, it is the synergy of both components renders the apparent power enhancement of the $\mathrm{LiFePO}_{4}$ component.

This synergy between the constituents in the hybrid electrode enhances the power and energy characteristics as well as significantly improves the cycling stability at high charge rates. The capacity retention plots (Fig. 3a) show that the hybrid electrode has enhanced rate performance than $\mathrm{LiFePO}_{4}$ and stores more charge than PTMA (see Supplementary information: Figs. S10 and S11 for electrochemical performance of other hybrid electrode configurations). Ultimately, the power and energy density of the hybrid electrode can be precisely balanced by the respective amount of constituents, to fulfill the targeted application requirements. Furthermore, the 


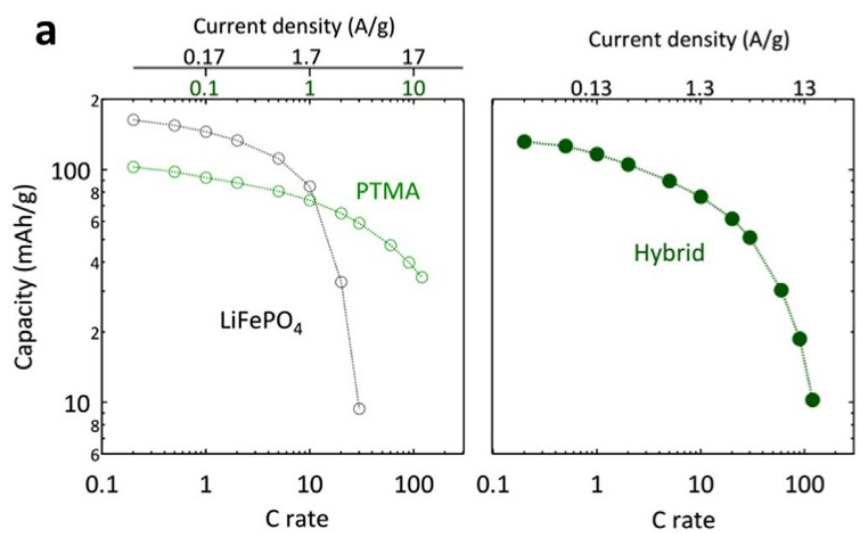

b

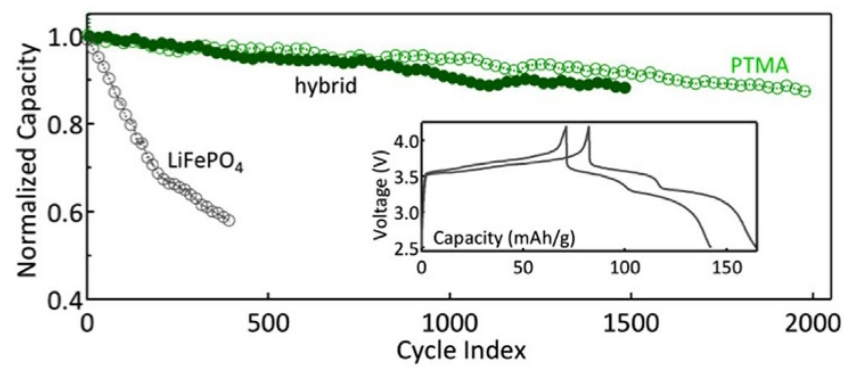

Figure 3 Electrochemical properties of the hybrid electrode.

(a), Capacity retention function of C-rate and applied current density (symmetrical charge - discharge conditions) for $\mathrm{LiFePO}_{4}, \mathrm{PTMA}$ and hybrid electrode. The green and black top axes correspond to PTMA and $\mathrm{LiFePO}_{4}$ respectively. The rate of $1 \mathrm{C}$ corresponds to 100,170 and $130 \mathrm{mAh} / \mathrm{g}$ for PTMA, LiFePO4 and hybrid electrode respectively. (b), Capacity retention at a charge - discharge rate of $5 \mathrm{C}$ for $\mathrm{LiFePO}_{4}$, PTMA and hybrid electrodes. Inset: voltage profiles for the hybrid electrode at $1^{\text {st }}$ and $1,000^{\text {th }}$ cycle.

hybrid electrode shows dramatic cycling stability enhancement at high recharge - discharge rates: only $12 \%$ capacity loss after 1,500 cycles at rate of $5 \mathrm{C}$. It is clear that the hybrid electrode has prolonged cycle life similar to the PTMA electrode rather than $\mathrm{LiFePO}_{4}$ (Fig. 3b). The voltage profiles for the hybrid electrode at the $1^{\text {st }}$ and $1,000^{\text {th }}$ cycle (inset to Fig. $3 \mathrm{~b}$ ) show that there is no preferential degradation of any of the constituents. It should be noted that here as well, the hybrid electrode configuration and composition strongly impact the electrode performance (see also supplementary information: figs. S12-S16 for details).

Fast charge storage mechanism. In addition to improved cycling and rate performances, we detail on a unique fast charge storage mechanism the hybrid electrode, which is schematized in Fig. 4a. The cyclic voltammetry analysis (Fig. 2) implies that the fast charge in the hybrid electrode is controlled by PTMA, i.e. limited polarization resulting in postponed $\mathrm{LiFePO}_{4}$ voltage rise and high charge transfer rate. When a charge current is applied on the hybrid electrode, the polarization of PTMA and $\mathrm{LiFePO}_{4}$ overlap above the equilibrium values and both components are charged (or, oxidized). However, the faster redox kinetics of PTMA results in excess charging of the PTMA component. When the current supply is stopped, the potential of both components in the electrode tends to reach their equilibrium open circuit potential. However, the electrochemical potential of the $\mathrm{PTMA} \mathrm{PTMA}^{+}$redox couple (3.63 V vs. $\left.\mathrm{Li} / \mathrm{Li}^{+}\right)$is higher than that of $\mathrm{LiFePO}_{4} / \mathrm{FePO}_{4}(3.44 \mathrm{~V}$ vs. $\left.\mathrm{Li} / \mathrm{Li}^{+}\right)$. The transient configuration is thus thermodynamically not favored and forces the spontaneous oxidation of $\mathrm{LiFePO}_{4}$ in the presence of $\mathrm{PTMA}^{+}$up to the complete consumption of the later. According to the first law of thermodynamics the overall state-of-charge (SOC) of the electrode will remain unchanged, mainly the PTMA/LiFePO 4 charged species ratio will change (see also Fig. 4c).

To better understand this process, we have monitored the relaxation voltage of the hybrid electrode charged at different rates and to different SOCs. Figure $4 \mathrm{~b}$ shows the open circuit potential (OCP) relaxation curves. After the initial voltage drop, a monotonic decay region is detected from $\sim 3,8 \mathrm{~V}$ to $\sim 3,6 \mathrm{~V}$, followed by an abrupt descent to $\sim 3,44 \mathrm{~V}$. From coulometric titration (supplementary information: Fig. S17) we identified the equilibrium OCPs in the hybrid electrode. Though the electrode is not at equilibrium, during the relaxation, the OCP value is proportional to the amount of $\mathrm{PTMA}^{+}$species (supplementary information: Figs. S17 and S18). After the complete consumption of $\mathrm{PTMA}^{+}$, a sudden OCP drop is noticed and the electrode potential stabilizes at the $\mathrm{LiFePO}_{4}$ equilibrium OCP $(3.44 \mathrm{~V})$. For $1: 1$ (capacity ratio) balancing of the electrode components, charging below $50 \%$ SOC will always result in an OCP relaxation drop at $3.44 \mathrm{~V}$, i.e., at equilibrium, $\mathrm{LiFePO}_{4}$ will be primarily charged (the 50\% SOC is schematized in Fig. 4a).

To further support this mechanism, we have analyzed the galvanostatic response of the hybrid electrode. Figure $4 \mathrm{c}$ shows the voltage profiles for the hybrid electrode charged at a rate of $45 \mathrm{C}$ and discharged at various rates. During the slow discharge $(0.2 \mathrm{C})$, mainly $\mathrm{LiFePO}_{4}$ contributes to the delivered capacity. The same situation is observed for the fully relaxed electrode (Figure 4b, 45 C, 44\% SOC). Augmenting the discharge rate $(0.35 \mathrm{C}-1.4 \mathrm{C})$ increases the PTMA contribution to the discharge at the expense of $\mathrm{LiFePO}_{4}$, whereas the total discharge capacity of the hybrid electrode is not found to vary significantly - confirming that the electrode SOC remains unchanged, and only the PTMA/LiFePO4 charged species ratio varies. Increasing further the discharge rate augments the relative contribution of PTMA to the total discharge, with a preponderant response of PTMA at high rates. The asymmetry in the charge discharge behavior is associated to the internal redox relaxation mechanism (see also supplementary information: Fig. S19). The discharge process is concurrent with the internal redox relaxation. Hence, PTMA has two possible routes for reduction: either by transferring the charge through the external circuit or by spontaneously reacting with $\mathrm{LiFePO}_{4}$. For example, after the $45 \mathrm{C}$ current pulse, the full relaxation in the hybrid electrode is reached within approximately 5 minutes (Fig. 4b). On the other hand, the time required to discharge the respective electrode at a rate of $0.2 \mathrm{C}$ is approximately 2 hours. The time scale difference is significative, meaning that the PTMA will preferentially follow the internal redox relaxation route. At higher discharge rate however, the duration required to discharge the electrode becomes comparable or even lower than the internal redox relaxation duration and PTMA dominates the discharge voltage profile.

The internal redox relaxation involving PTMA discharge (reduction) and $\mathrm{LiFePO}_{4}$ charge (oxidation) is favored by the hybrid electrode configuration (supplementary information: Fig. S18). The intimate contact between the components ensures an efficient electron transfer and ionic diffusion interface during the relaxation reaction. In fact, $\mathrm{LiFePO}_{4}$ can support fast redox reaction, consistent with high $\mathrm{Li}^{+}$diffusivity found in single particle experiments and using first principle methods $s^{34,46}$. Moreover, when reduced, PTMA liberates $\mathrm{PF}_{6}{ }^{-}$counter-ions in the electrolyte, whereas $\mathrm{LiFePO}_{4}$ releases $\mathrm{Li}^{+}$ions when oxidized. During the relaxation, both reactions occur with a perfect stoichiometry. The nanometer separation between the reaction sites in the hybrid electrode ensures fast equilibration of ionic species. Different hybrid configurations also display the internal redox equilibration process however, with different time constant owing to the spatial separation of the constituent materials (Supplementary information: Fig. S18). This should help further design of enhanced hybrid electrodes and better understand the redox and diffusion processes in battery electrodes at nanoscale. 


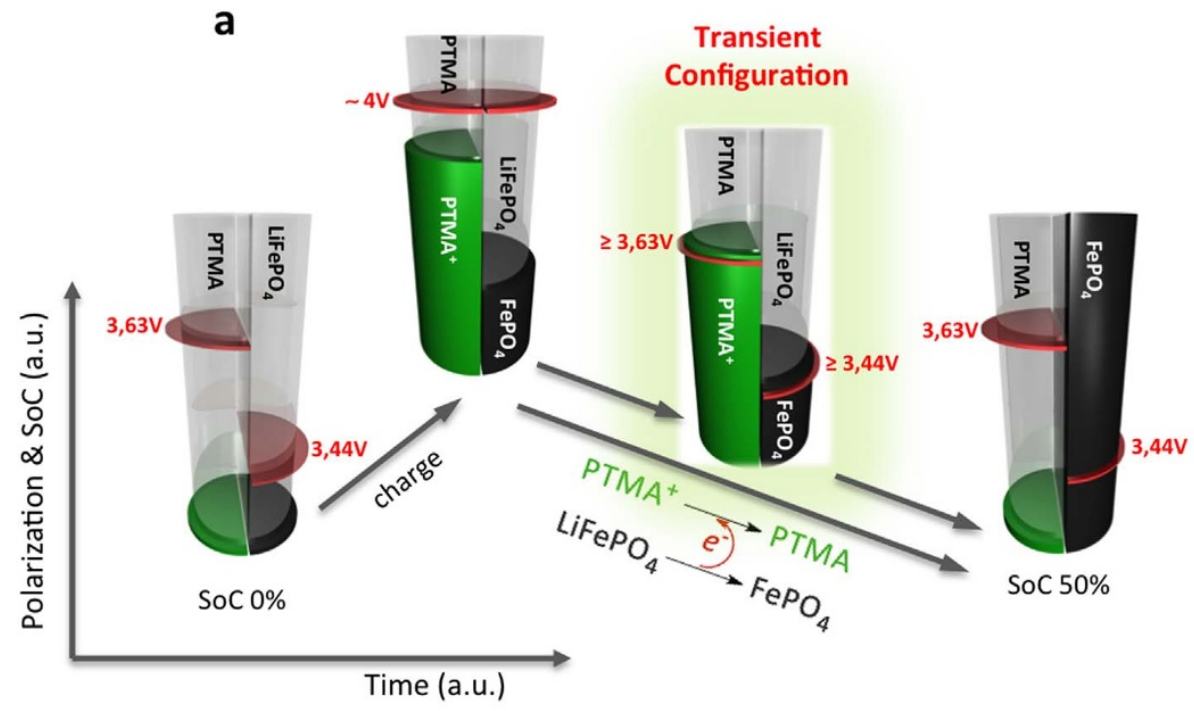

b

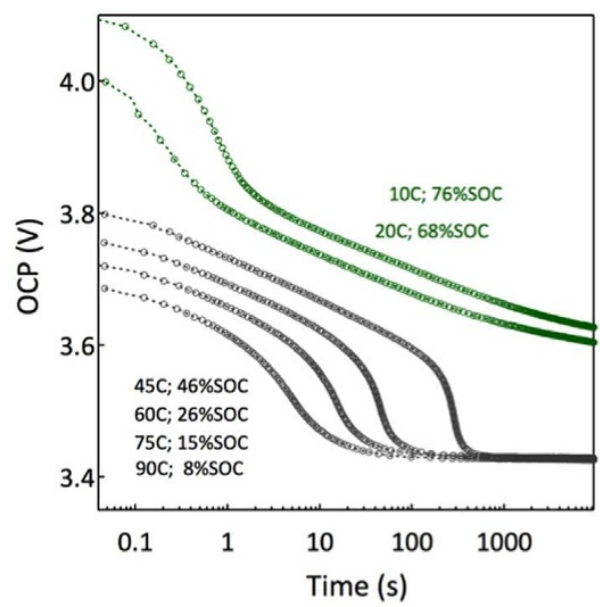

C

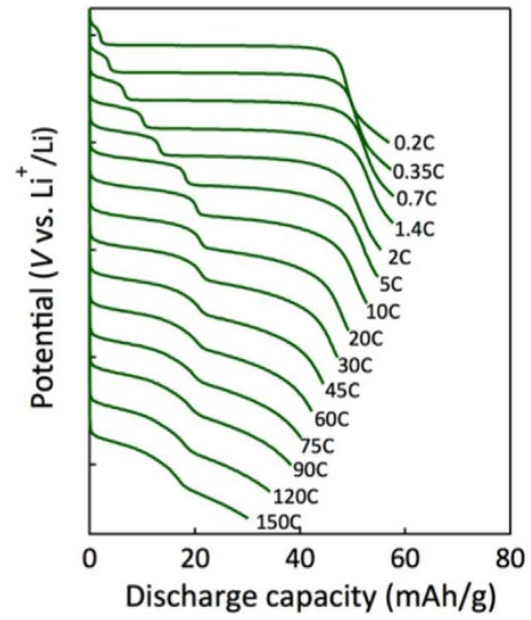

Figure $4 \mid$ Charge storage mechanism in the hybrid electrode. (a), Schematic representation of the fast charge - relaxation process. The green and black half-cylinders are indicative of the stored charge in $\mathrm{PTMA}_{\text {and }} \mathrm{LiFePO}_{4}$ respectively. The relative position of the red disk indicates the polarization of the constituents in the hybrid electrode. Except during the charge, the polarization is actually the equilibrium OCP for each component. Fast charging leads to a thermodynamically unstable configuration, characterized by evanescent co-existence of high potential $\mathrm{PTMA}^{+}$with low potential LiFePO ${ }_{4}$ species forcing an internal charge transfer equilibration. The depicted situation is representative of $50 \% \mathrm{SOC}$, i.e., at equilibrium, only the $\mathrm{LiFePO}_{4}$ component will be charged. (b), Relaxation curves after charging the hybrid electrode at different rates and to different SOC. (c), Voltage profile at different discharge rates (the curves are shifted for clarity). For every discharge, the charge rate was $45 \mathrm{C}$.

Fast battery charging via relaxation control. Since fast charging of batteries is utmost important for widespread adoption of electric vehicles we studied the hybrid electrode performance under fast charging conditions. An exciting feature of the hybrid electrode is that the difference in the redox potentials between the hybridized materials will always lead, at equilibrium, to the preferential charging of $\mathrm{LiFePO}_{4}$ at the expense of PTMA. Nonetheless, PTMA is the faster recharge component resulting in a highly relevant practical fact: whenever the electrode needs to be recharged, the rapid response of PTMA will ensure the fast recharge. A combination of pulse charge and relaxation of PTMA-LFP hybrid electrode was studied to estimate the rapid charging protocol and was found to lead to elevated electrode SOC in short time intervals. Interestingly, the SOC can be controlled here by adjusting the relaxation time. A typical sequence is shown in Figure 5a (see also supplementary information: Figs. S20-S22) consisting in the application of consecutive current pulses of $30 \mathrm{C}, 10 \mathrm{C}$ and $3 \mathrm{C}$, with different relaxation periods in between. For each pulse, different charge rates have been used here in order to maintain the same current density experienced by the remaining uncharged material. After the first current pulse (30 C charge) both electrodes reach approximately $63 \%$ SOC. The charge voltage profiles are similar and the ratio of redox species are expected to be identical by the end of the first charge sequence. It is the duration of the subsequent relaxation step that induces the difference. If the internal redox equilibration is allowed to occur for 30 minutes (top plot), during the second charge sequence ( $10 \mathrm{C}$ charge), the average voltage is $3.75 \mathrm{~V}$ and the attained SOC is close to $90 \%$. Whereas if only 30 seconds relaxation is allowed (bottom panel) a lower SOC is reached $(81 \%)$ and the average charge voltage is higher $(3.85 \mathrm{~V})$. Clearly, the availability of uncharged PTMA (or the amount of regenerated PTMA) positively impacts the fast recharge capability of the hybrid electrode. The same behavior is observed for the last relaxation - charge sequence: higher SOC at lower charge voltage and lower SOC at higher voltage and for 30 minutes and 10 seconds of relaxation time respectively.

The apparent charging time for both situations is one hour and 5 minutes resulting in approximately $96 \%$ and $90 \%$ SOC respectively. 

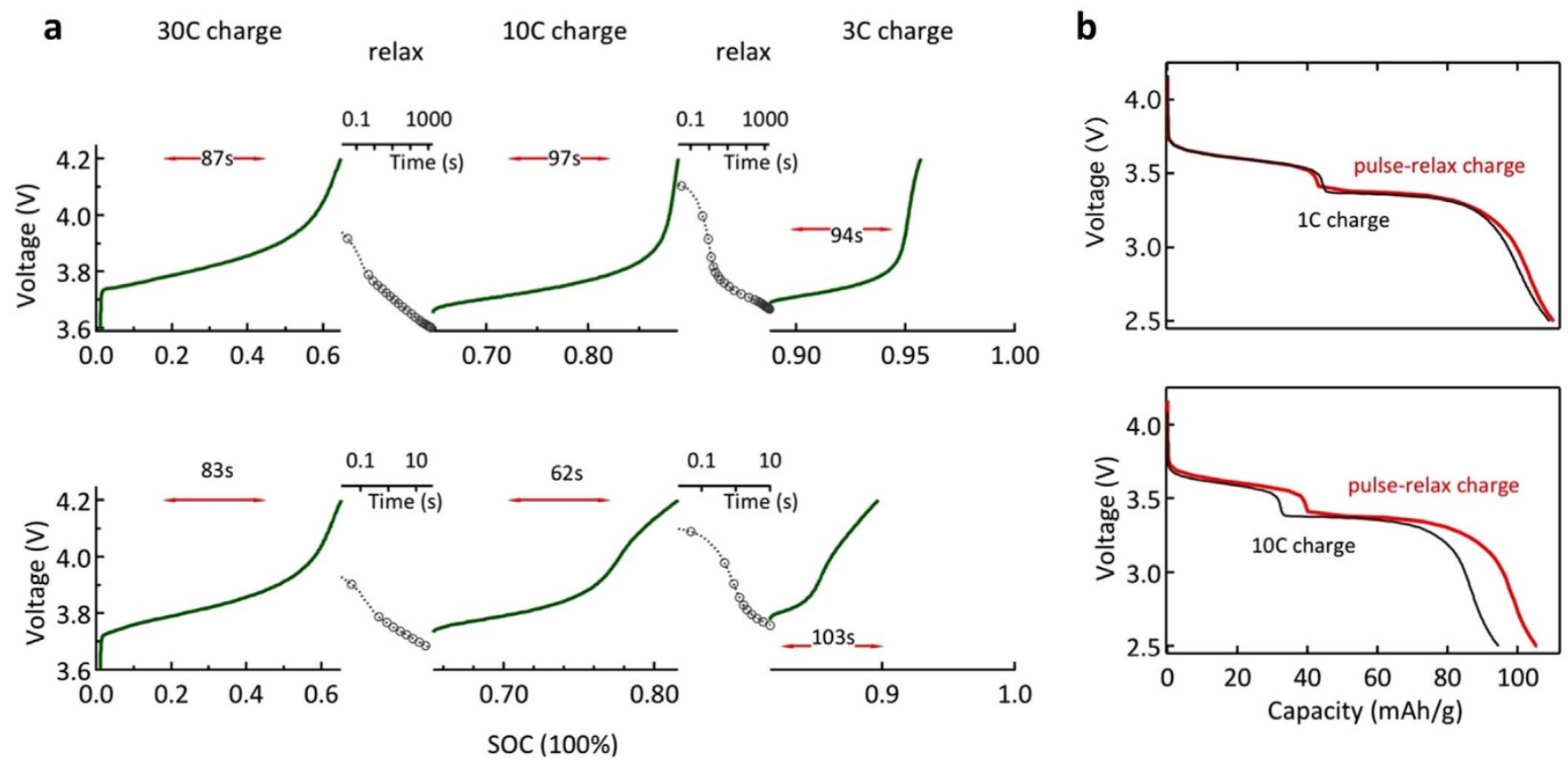

Figure 5 | Fast recharge of the hybrid electrode. (a), Voltage profile during charge (green) and relaxation (gray) sequences. Top panel: relaxation periods of 30 minutes; bottom panel: 30 and 10 seconds. The effective charge duration for each step is highlighted. The apparent charge time is 1 hour and 5 minutes respectively. (b), Corresponding discharge profiles (red curves, rate of $1 \mathrm{C}$ ) as well as the discharge profile for constant-current charge mode for an equivalent period of time, 1 hour and $\sim 5$ minutes respectively (grey curves, rate of $1 \mathrm{C}$ ).

Alluring, charging in constant current mode for an equivalent period of time ( $\sim 5$ minutes at a charge rate of $10 \mathrm{C})$, results in a lower SOC $(\sim 80 \%)$ in the hybrid battery electrode (Fig. 5 b). For the one-hour charge situation, no much difference is noticed between the two charge modes. It is noteworthy the impact of the relaxation process on the recharge performance of the hybrid electrode especially if fast recharge is intended. The ability of PTMA to absorb high current loads, operating as an input power buffer and subsequently, delivering the stored charge to the high-energy component, illustrates a new class of hybrid electrodes with fast charge storage capability through simple, user defined protocols.

Due to the higher redox potential of PTMA as compared to $\mathrm{LiFePO}_{4}$, the present hybrid configuration is primarily intended to enhance the high recharge capability of the electrode. Power delivery of the hybrid electrode is also enhanced due to higher rate performance of PTMA. However, the intra-electrode redox synergy is unfavorable as the redox species equilibration will result in exhaustion of charged $\mathrm{PTMA}^{+}$that will affect the subsequent discharge pulses (supplementary information: Fig. S16). Nevertheless, following this rationale, coupling the PTMA with a higher voltage cathode material will result in enhance power delivery characteristics, as the charged $\mathrm{PTMA}^{+}$will be the favored redox species in the hybrid electrode, capable to sustain high discharge rates. Furthermore, the redox potential of PTMA could be readily tuned by altering the molecular environment of the nitroxide group making possible the realization of higher voltage nitroxide supercapacitors ${ }^{47,48}$. Such molecularly engineered architectures could be further coupled to other cathode materials than $\mathrm{LiFePO}_{4}$, targeting the redox synergy in either rapid charge or discharge, extending thus the applicability of the presented hybridization scheme.

The hybridization of the two separate components yields a remarkable set of properties. The appropriate redox couples, flatpotential profile and elevated specific capacity yet, different redox kinetics for PTMA and $\mathrm{LiFePO}_{4}$, offer a hybrid battery electrode where the fast electrochemical response of PTMA delays the voltage rise during the charge process. This implies significant improvements for the rate performance, cycle lifetime and safety of lithium-ion batteries during rapid recharge. Proper balancing of both constituents could lead to a large variety of electrode formulations with user designed performances: more $\mathrm{LiFePO}_{4}$ for high energy density applications at the expense of power energy density, whereas the addition of PTMA will result in enhanced power capabilities with a lower decrease in the energy density. The hybrid electrode is expected to play a significant role for power leveling in solar conversion - storage units, peak supply and demand for better grids or fast recharge of EV's ${ }^{49}$. This novel approach paves the way to new design rules for Li-ion battery electrodes and may prove pivotal in pushing the performance envelope of Li-ion batteries towards the goal of increasing adoption of electric vehicles. Clearly, this unconventional hybridization does not result here in a simple addition of the characteristic features of both components as in the classical case of EDLC and battery materials hybridization.

1. Tarascon, J. \& Armand, M. Issues and challenges facing rechargeable lithium batteries. Nature 414, 359-367 (2001).

2. Armand, M. \& Tarascon, J. M. Building better batteries. Nature 451, 652-657 (2008).

3. Choi, N.-S. et al. Challenges facing lithium batteries and electrical double-layer capacitors. Angew. Chem. Intl. Ed. 51, 9994-10024 (2012).

4. van Schalkwijk, W. A. \& Scrosati, B. Advances in Lithium-Ion Batteries (Kluwer Academic/Plenum 2002).

5. Zhang, H., Yu, X. \& Braun, P. Three-dimensional bicontinuous ultrafast-charge and -discharge bulk battery electrodes. Nat. Nanotech. 6, 277-281 (2011).

6. Kang, B. \& Ceder, G. Battery materials for ultrafast charging and discharging. Nature 458, 190-193 (2009).

7. Vlad, A. et al. Roll up nanowire battery from silicon chips. Proc. Natl. Acad. Sci. 109, 15168-15173 (2012).

8. Singh, N. et al. Paintable battery. Sci. Rep. 2, 481 (2012).

9. Wang, J.et al. Nature of $\mathrm{LiFePO}_{4}$ aging process: Roles of impurity phases. J. Power Sources 238, 454-463 (2013).

10. Dubarry, M. \& Liaw, B. Y. Identify capacity fading mechanism in a commercial $\mathrm{LiFePO}_{4}$ cell. J. Power Sources 194, 541-549 (2009).

11. Kao, Y.-H. et al. Overpotential-Dependent Phase Transformation Pathways in Lithium Iron Phosphate Battery Electrodes. Chem. Mater. 22, 5845-5855 (2010).

12. Wang, Y., Zhao, D., Che, R. \& Xia, Y. Pseudo-capacitive profile vs. Li-intercalation in Nano-LiFePO 4 . J. Power Sources 236, 230-237 (2013).

13. Goodenough, J. B. \& Park, K.-S. The Li-Ion Rechargeable Battery: A Perspective. J. Am. Chem. Soc. 135, 1167-1176 (2013). 
14. Simon, P. \& Gogotsi, Y. Materials for electrochemical capacitors. Nat. Mater. 7, 845-854 (2008).

15. Novak, P., Muller, K., Santhanam, K. \& Haas, O. Electrochemically active polymers for rechargeable batteries. Chem. Rev. 97, 207-281 (1997).

16. Lang, X., Hirata, A., Fujita, T. \& Chen, M. Nanoporous metal/oxide hybrid electrodes for electrochemical supercapacitors. Nat. Nanotech. 6, 232-236 (2011).

17. Augustyn, V. et al. High-rate electrochemical energy storage through $\mathrm{Li}(+)$ intercalation pseudocapacitance. Nat. Mater. 12, 518-522 (2013).

18. Cericola, D. \& Kötz, R. Hybridization of rechargeable batteries and electrochemical capacitors: Principles and limits. Electrochimica Acta 72, 1-17 (2012).

19. Du Pasquier, A., Plitz, I., Menocal, S. \& Amatucci, G. A comparative study of Liion battery, supercapacitor and nonaqueous asymmetric hybrid devices for automotive applications. J. Power Sources 115, 171-178 (2003).

20. Chen, S. et al. ( $\left(\mathrm{LiFePO}_{4}-\mathrm{AC}\right) / \mathrm{Li}_{4} \mathrm{Ti}_{5} \mathrm{O}_{12}$ hybrid supercapacitor: The effect of $\mathrm{LiFePO}_{4}$ content on its performance. J. Renewable Sustainable Energy 4, 033114 (2012).

21. Naoi, K., Ishimoto, S., Miyamoto, J.-I. \& Naoi, W. Second generation 'nanohybrid supercapacitor': Evolution of capacitive energy storage devices. Energy Environ. Sci. 5, 9363 (2012)

22. Choi, H. S., Im, J. H., Kim, T., Park, J. H. \& Park, C. R. Advanced energy storage device: a hybrid BatCap system consisting of battery-supercapacitor hybrid electrodes based on $\mathrm{Li}_{4} \mathrm{Ti}_{5} \mathrm{O}_{12}$-activated-carbon hybrid nanotubes. J. Mater. Chem. 22, 16986 (2012).

23. Huang, Y.-H. \& Goodenough, J. B. High-Rate $\mathrm{LiFePO}_{4}$ Lithium Rechargeable Battery Promoted by Electrochemically Active Polymers. Chem. Mater. 20, 7237-7241 (2008).

24. Huang, Q., Cosimbescu, L., Koech, P., Choi, D. \& Lemmon, J. P. Composite organic radical-inorganic hybrid cathode for lithium-ion batteries. J. Power Sources 233, 69-73 (2013).

25. Chen, Y., Freunberger, S. A., Peng, Z., Fontaine, O. \& Bruce, P. G. Charging a Li$\mathrm{O}_{2}$ battery using a redox mediator. Nat. Chem. 5, 489-494 (2013).

26. Weng, W. et al. Smart Polymeric Cathode Material with Intrinsic Overcharge Protection Based on a 2,5-Di-tert-butyl- 1,4-dimethoxybenzene Core Structure. Adv. Funct. Mater. 22, 4485-4492 (2012).

27. Huang, X. et al. Charge rate influence on the electrochemical performance of $\mathrm{LiFePO}_{4}$ electrode with redox shuttle additive in electrolyte. Ionics 18, 501-505 (2012)

28. Wang, Q. et al. Thermal runaway caused fire and explosion of lithium ion battery. J. Power Sources 208, 210-224 (2012).

29. Wang, Q., Evans, N., Zakeeruddin, S. M., Exnar, I. \& Grätzel, M. Molecular wiring of insulators: charging and discharging electrode materials for high-energy lithium-ion batteries by molecular charge transport layers. J. Am. Chem. Soc. 129, 3163-3167 (2007)

30. Badway, F. et al. Structure and electrochemistry of copper fluoride nanocomposites utilizing mixed conducting matrices. Chem. Mater. 19, 4129-4141 (2007)

31. Nishide, H. \& Suga, T. Organic radical battery. The Electrochemical Society Interface Winter, 32-36 (2005).

32. Suga, T., Pu, Y.-J., Oyaizu, K. \& Nishide, H. Electron-Transfer Kinetics of Nitroxide Radicals as an Electrode-Active Material. Bull. Chem. Soc. Jpn. 77, 2203-2204 (2004)

33. Nakahara, K. et al. High-rate capable organic radical cathodes for lithium rechargeable batteries. J. Power Sources 165, 870-873 (2007).

34. Malik, R., Burch, D., Bazant, M. \& Ceder, G. Particle size dependence of the ionic diffusivity. Nano Lett. 10, 4123-4127 (2010).

35. Chung, S.-Y., Bloking, J. T. \& Chiang, Y.-M. Electronically conductive phosphoolivines as lithium storage electrodes. Nat. Mater. 1, 123-128 (2002).

36. Wang, L. et al. Crystal Orientation Tuning of $\mathrm{LiFePO}_{4}$ Nanoplates for High Rate Lithium Battery Cathode Materials. Nano Lett. 12, 5632-5636 (2012).
37. Herle, P. S., Ellis, B., Coombs, N. \& Nazar, L. F. Nano-network electronic conduction in iron and nickel olivine phosphates. Nat. Mater. 3, 147-152 (2004).

38. Park, O. K. et al. Who will drive electric vehicles, olivine or spinel? Energy Environ. Sci. 4, 1621-1633 (2011).

39. Zaghib, K. et al. Review and analysis of nanostructured olivine-based lithium recheargeable batteries: Status and trends. J. Power Sources 232, 357-369 (2013).

40. Bi, Z., Zhang, X., He, W., Min, D. \& Zhang, W. Recent advances in $\mathrm{LiFePO}_{4}$ nanoparticles with different morphology for high-performance lithium-ion batteries. RSC Adv. 3, 19744-19751 (2013).

41. Kim, J.-K. et al. Nano-fibrous polymer films for organic rechargeable batteries. J. Mater. Chem. A 1, 2426 (2013).

42. Guo, W., Yin, Y.-X., Xin, S., Guo, Y.-G. \& Wan, L.-J. Superior radical polymer cathode material with a two-electron process redox reaction promoted by graphene. Energy Environ. Sci. 5, 5221 (2012).

43. Choi, W., Ohtani, S., Oyaizu, K., Nishide, H. \& Geckeler, K. E. Radical PolymerWrapped SWNTs at a Molecular Level: High-Rate Redox Mediation Through a Percolation Network for a Transparent Charge-Storage Material. Adv. Mater. 23, 4440-4443 (2011).

44. Ouvrard, G. et al. Heterogeneous behaviour of the lithium battery composite electrode $\mathrm{LiFePO}_{4}$. J. Power Sources 229, 16-21 (2013).

45. Lindström, H. et al. $\mathrm{Li}^{+}$ion insertion in $\mathrm{TiO}_{2}$ (anatase). 2. Voltammetry on nanoporous films. J. Phys. Chem. B 101, 7717-7722 (1997).

46. Kanamura, H., Takemura, B., Saito, T. \& Kanamura, K. Evaluation of real performance of LiFePO4 by using single particle technique. J. Power Sources 217, 444-448 (2012)

47. Suga, T., Pu, Y.-J., Kasatori, S. \& Nishide, H. Cathode- and anode-active poly(nitroxylstyrene)s for rechargeable batteries: $\mathrm{p}$ - and n-type redox switching via substituent effects. Macromol. 40, 3167-3173 (2007).

48. Bilgiç, B., Kılıç, C.. \& Esat, B. First-principles study of polyacetylene derivatives bearing nitroxide radicals. Phys. Rev. B 84, 115207 (2011).

49. Dunn, B., Kamath, H. \& Tarascon, J.-M. Electrical energy storage for the grid: A battery of choices. Science 334, 928-935 (2011).

\section{Acknowledgments}

A.V. and S.M. acknowledge F.R.S.-FNRS for financial support. J.R., S.M. and J.F.G. acknowledge financial support from Région Wallonne (Programme ERABLE). Work in Louvain la Neuve was partly supported by the ARC project 09/14-023 (Communauté Française de Belgique). We thank C. Galande for helpful discussions. N.S. and P.M.A. acknowledge Advance Energy Consortium for financial support.

\section{Author contributions}

A.V. conceived the idea and designed the experiments. A.V. carried out most of the experimental work. N.S. and J.R. contributed new reagents/analytic tools. A.V., N.S., J.R., S.M., P.A. and J.G. discussed the results and wrote the manuscript.

\section{Additional information}

Supplementary information accompanies this paper at http://www.nature.com/ scientificreports

Competing financial interests: UCL has filed a patent on some of the intellectual property disclosed by this paper.

How to cite this article: Vlad, A. et al. Hybrid supercapacitor-battery materials for fast electrochemical charge storage. Sci. Rep. 4, 4315; DOI:10.1038/srep04315 (2014).

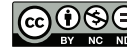

This work is licensed under a Creative Commons Attribution-

NonCommercial-NoDerivs 3.0 Unported license. To view a copy of this license, visit http://creativecommons.org/licenses/by-nc-nd/3.0 